\title{
On the warning system of obstacle avoidance of embedded electronic guide dogs
}

\author{
YUE Xiangyu \\ School of Management and Engineering, Nanjing University; Nanjing Jiangsu; 210093; PR China
}

\author{
Email address: \\ yuexiangyu168@gmail.com
}

\section{To cite this article:}

YUE Xiangyu. On the Warning System of Obstacle Avoidance of Embedded Electronic Guide Dogs. Automation, Control and Intelligent Systems. Vol. 1, No. 5, 2013, pp. 99-102. doi: 10.11648/j.acis.20130105.11

\begin{abstract}
With the rapid development of China's transportation, the frequency of traffic accidents is also high. This not only restricted the development of China's transportation greatly, but also threatened to people's safety seriously. In particular, the accidents caused by the blind due to there are more frequent reproduction, so their traffic safety has become a big issue to solve urgently. In the program, a new type of "warning system of obstacle avoidance of embedded electronic guide dog" has been developed on the basis of careful analysis of all kinds of present anti-collision warning systems, which has a core micro-controller, 32-bit ARM7 microprocessor, and takes the embedded operating system uCLinux as its platform. Such warning system of obstacle avoidance of embedded electronic guide dog can effectively eliminate the impact of the traffic environment and the subjective factors of the blind, warning in advance for the travelling blind in time, effectively avoiding obstacles such as vehicles, to reduce traffic accidents caused by the their blindness. This humane technology innovation is the specific embodiment of environmental science and technology aesthetic theory in the field of scientific and technological innovation. It has a positive and promoting role to the development of transportation and blind-man welfare in China.
\end{abstract}

Keywords: Embedded System, Electronic Guide Dog, Avoiding Obstacles, Warning, ARM, USB Communication, Uclinux

\section{Introduction}

During last summer, I went blind orphanage as a volunteer, and personally experienced the inconvenience and hardship of the blind when they travel. Especially when I heard the news that a blind man had been hit and killed when crossing the street just several days before because of his blindness, my compassion was once again inspired, and I was very eager to do my best to help the blind. I think that if electronic guide dogs guide them to avoid obstacles such as vehicles, the blind people are able to avoid such accidents, aren't they? So I had an idea that I use the scientific knowledge of embedded system grasped by me to invent a kind of "electronic guide dog". After that I began to design the structure of the entire system, which made the overall implementation of "electronic guide dog" take a welcome step.

In fact, this "warning system of embedded electronic guide dog avoiding obstacles" is similar to a car anti-collision avoidance system. Currently, some of the international anti-collision avoidance system development has made some achievements, and the more successful countries are Japan, Germany, and the United States. In China, the research and development in this area have relatively large gap with developed countries, and not until recent years did a number of research institutes and universities begin to study it. The research on "warning system of embedded electronic guide dog avoiding obstacles" in this paper is an important part of intelligent electronic guide dog system, the study of which is mainly based on the embedded system, anti-collision warning system, and the USB technology is applied to warning system of embedded electronic guide dog avoiding obstacles.

\section{The Design of Embedded Electronic Guide Dog of Obstacle-Avoiding Early Warning System}

The design of obstacle-avoiding early warning system of embedded electronic guide $\operatorname{dog}$ is an inheritance and innovation, based on the design of traffic information collecting system, which is generally used in vehicle 
anti-collision at present. Nowadays, the main types of the design of traffic information collecting system, generally used in vehicle anti-collision, are shown as follows, that is, using single chip microcomputer for data analysis, PC-centric (industrial control computer), using DSP for data analysis and so on. ${ }^{[1]}$ In the design process of the obstacle-avoiding early warning system of embedded electronic guide dog, we firstly has carried on a comprehensive evaluation and analysis for each scheme, and then put forward a new idea that it introduces the open source embedded real-time operating system uCLinux, with a high-powered ARM core as the core processor. ${ }^{[2]}$ We take this as the foundation, expanding a variety of the functions required, for instance, the function of USB communication interface, and human-machine interface. In this way, the design of obstacle-avoiding early warning system of embedded electronic guide $\operatorname{dog}$ has the following advantages. It has a simple design, and small volume, so that it is convenient to carry the "dog". Also, it is easy to extend the functions, which is convenient for field operations, greatly reducing the cost of the traffic information collecting system in the obstacle-avoiding early warning system of electronic guide dog.

What's more, the design of obstacle-avoiding early warning system of embedded electronic guide dog has utilized the core processor, based on the realization of the system function, and development conditions. Its operating system is thought from the perspectives of practical application, development tool, instantaneity, technical services, and price, etc. Its main control unit hardware uses the ARM microprocessor as the core, processing and handling the signal of each sensor. ${ }^{[3]}$ The alarm system of guide dog adopts the combination of light and sound. Sound will produce auditory stimulus for the blind. Meanwhile, light and sound may make an auditory and visual stimulation for other pedestrians, arousing the attention of the blind and pedestrians to let them take appropriate actions at the same time to avoid accidents. Moreover, the obstacle-avoiding early warning system of embedded electronic guide dog also selects USB protocol to transfer data, saving the collected data in the hardware after being managed.

The overall design plan of obstacle-avoiding early warning system of embedded electronic guide dog mainly is the velocity and distance measurement module, the power circuit module, the LED screen show module, USBcommunication circuit module, and clock circuit module. The hardware of the obstacle-avoiding early warning system of embedded electronic guide dog can reference the Figure 1:

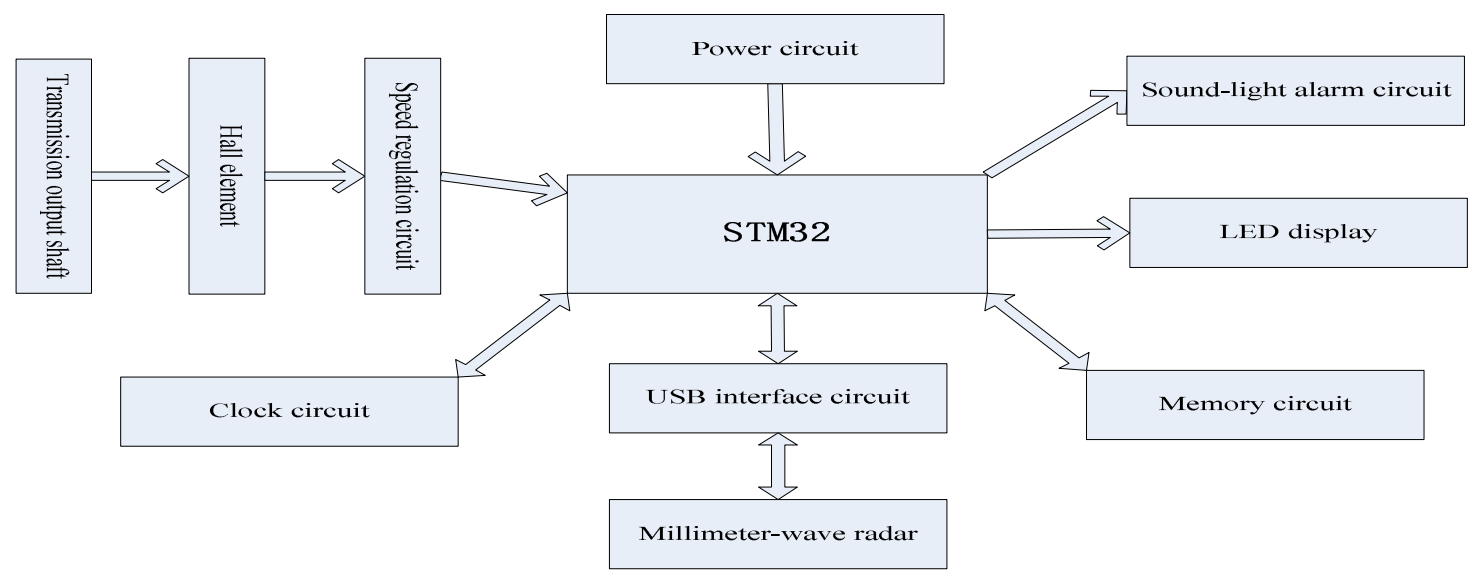

Figure 1: Schematic diagram of hardware system structure ${ }^{[4]}$

\section{The Application of Embedded Electronic Guide Dog of Obstacle-Avoiding Early Warning System}

In the anti-collision early warning system, referenced in the design of obstacle-avoiding early warning system of embedded electronic guide dog, there mainly exits four steps, that is, information collection, information processing, information judgment, and warning information. The techniques of information collection are ultrasonic wave, laser, infrared ray, machine vision and interactive method. Information processing is mainly to analyze the collected information, usually using ARM, MCU and DSP microprocessor, etc. ${ }^{[5]}$ And information judgment is based on the information, like the model of the guide dog, opportunity, weather, the distance between the guide dog and the obstacle, relative acceleration, relative speed and so on. Through the technique of information fusion, this step will make a real-time dynamic measurement and identification of its risk or safety state.

Presently, the types of the generally used anti-collision warning systems mainly are radar anti-collision warning system, ultrasonic anti-collision warning system, laser anti-collision warning system, infrared anti-collision warning system, machine vision anti-collision warning system, and interactive intelligent anti-collision warning system. The selection of anti-collision warning system for the design of obstacle-avoiding early warning system of embedded electronic guide dog should start from the 
characteristics of the highway network and the street network construction in our country, combing with the characteristics of the obstacle-avoiding early warning system of electronic guide dog, as well as the construction of our country's highway and street traffic integrated management system. We should optimize the choice of the obstacle-avoiding early warning system technique of embedded electronic guide dog, based on China's national situation. Therefore, in the design process of the obstacle-avoiding early warning system of embedded electronic guide dog, after the comprehensive and comparative analysis of the anti-collision warning techniques, we have compared the optimization principles of the anti-collision warning techniques, and finally adopt the approach of millimeter wave radar sensor + ARM microprocessor.

The design of obstacle-avoiding early warning system of embedded electronic guide dog also adopts the generally used embedded processor. Presently, the generally used embedded processors mainly are ARM, Power PC, MIPS, Motorola 68000 series, etc. The full name of ARM is Advanced RISC Machines. The ARM architecture follows the principle of reduced instruction set computer (RISC). It has a variety of merits, like small size, high performance, low power consumption, and cheap cost. It extensively uses the registers with a fast speed of instruction execution. And its way to addressing is much easier and more flexible, and its operating efficiency is high. Also, it could complete most of the data manipulation in the register. Due to the operating system is an aggregation of the system program, people don't have to consider the differences between different hardware, and could achieve the target of writing application software through the system interface provided uniformly. The embedded system will further compile all of its procedure codes, including the operating system code, driver's code and application code, into a whole paragraph of executable code and in inserted them into the hardware. In this way, the embedded operating system in embedded system is more like a set of function library. And the embedded operating system should satisfy basic requirements of the reliability, real-time performance, and tailing capability. At present, the embedded RTOS (Real Time Operate System) mainly includes two categories--commercial and free. Thereinto, the commercial RTOS includes WINCE and VxWotks; while the free RTOS has Linux (uCLinux and RT Linux included) and uC/OS - II. UCLinux aimed at the micro-control field, designing the Linux system, which is specially designed for the CPU without MMU (such as the S3C44B0X adopted in this project), and it has done a lot miniaturization work for the Linux kernel. These are the most remarkable features that uCLinux owned. Besides, its other main features are universal Linux API, core file less than $512 \mathrm{~KB}$, core+file system less than $900 \mathrm{~KB}$, and complete TCP/IP. Also, it supports a large number of other network protocols and various file systems, including NFS, EXT2, ROMFS, JFFS, ms-dos, FAT16/32, etc.

In addition, the design of obstacle-avoiding early warning system of embedded electronic guide dog also makes a full use of USB. USB is a new kind of computer peripheral communication interface standard, which abandons the defects of the traditional computer series/parallel, with advantages of the reliability of data transmission, hot plug (plug and play), convenient extension, and low cost. The reference model is shown in Figure 2.
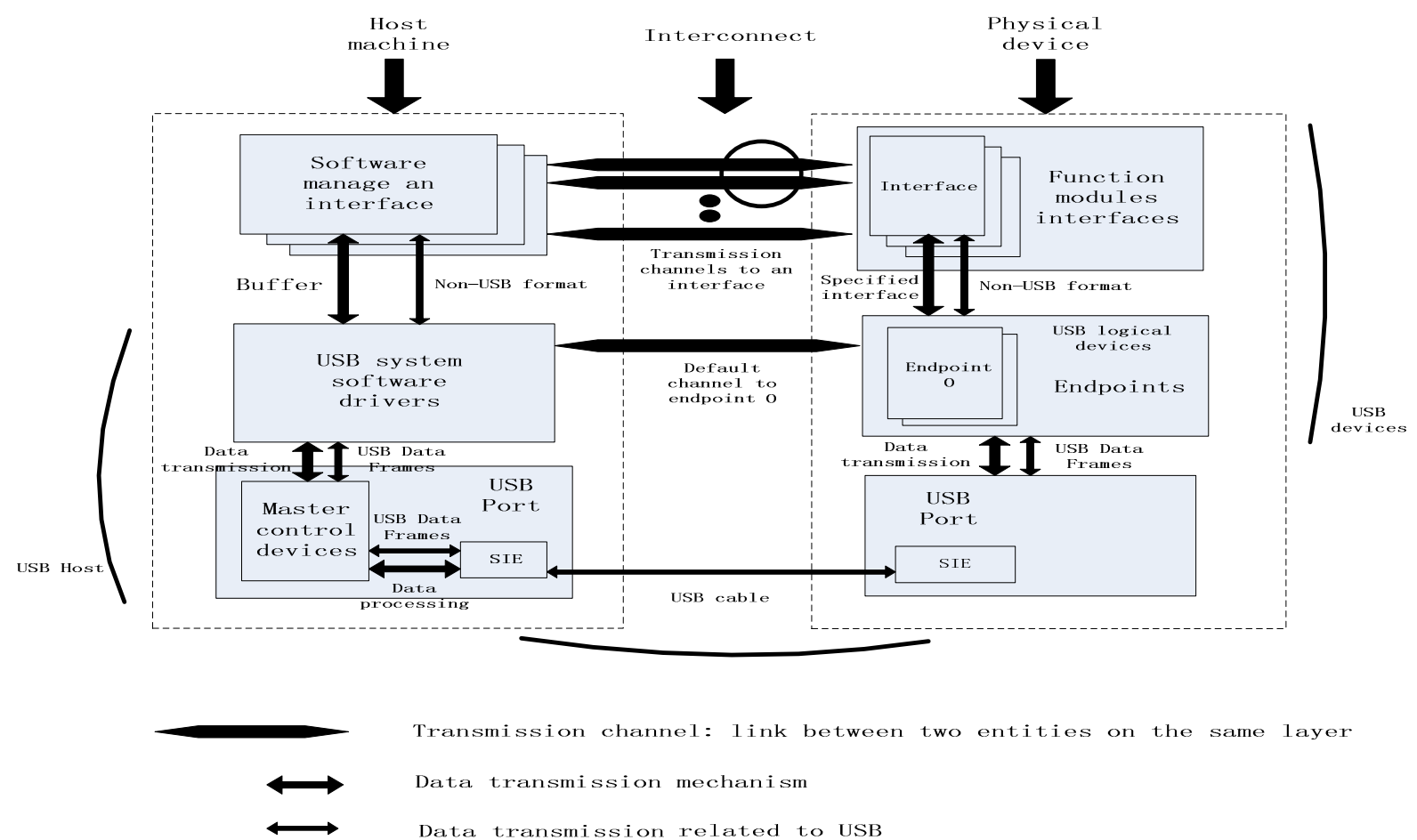

Figure 2: the structure diagram of USB reference model ${ }^{[4]}$ 


\section{Conclusion}

Today, with the rapid development of transport and vehicles, the frequency of traffic accidents has been being high, which not only greatly restricted the development of transport, but also made people travel in great danger. The traffic safety problem of the blind is an urgent issue to solve. The warning system of embedded electronic guide dog avoiding obstacles studied in the project can effectively eliminate the influence of traffic environment and blind subjective factors, send the pre-trip alarm for the blind in time, effectively avoid obstacles such vehicles, to reduce traffic accidents caused due to the blindness. This humanized technology innovation is the embodiment of environmental science and technology aesthetics theory in the field of science and technology innovation practice. This has not only a positive role in promoting the development of Chinese transport and the blind welfare, but also important practical significance and practical value for us to build a harmonious society and a beautiful China.

\section{Brief Introduction to the Author}

Yue Xiangyu (1992- ), who is a male, Han nationality, was born in Weifang, Shandong, with BA, Nanjing University, mainly engaged in electrical information and automation, aesthetics and other aspects of learning and research, specializing in modern industrial embedded control systems, networked control systems, intelligent control. He presided over one national college student science and technology innovation project, taking part in the research on one "Eleventh Five-Year Plan" education and science key project of the Education Ministry, and one soft science research project of Shandong Province. He has 12 science and technology papers published in Chinese and oversea academic journals. He has won the 1st scholarship of China, the 1st scholarship of people, the 1st and the 2nd prizes of China Education Robotics Competition, the 1st prize of China Mathematical Modeling Contest, the top award of scientific research achievement of Nanjing University, two China science and technology patents and the honorary title, "three-good-student" of Jiangsu Province. In addition, as a representative of Nanjing University, he went to the National University of Singapore to participate in an academic exchange program. Address: School of Management and Engineering, Gulou Campus of Nanjing University, 22, Hankou Avenue, Gulou District, Nanjing, Jiangsu Province, PR China; Zip: 210093.

\section{References}

[1] R. Weil, Jwooton, A. Garcia-Ortiz. Traffic Incident Detection: Sensors and Algorithms [A]. Math Comput Modeling [J], 1998, (27): 257 - P291.

[2] Zhu Weiwei, Yang Jianming. uClinux: an embedded Linux system[A]. Ship Electronic Engineering [J], 2003,12 (3): 3-5.

[3] GL Duckworth. A comparative study of traffic monitoring sensors [A]. Proceedings of the IVHS AMERICA annual meetingmAtlanta [C], 1994: 283-293.

[4] Ji Xiaojiang, Zhao Jing. Vehicle Rear Pre-Alarm System[A]. Enterprise technology development[J]. 2011, (12): 8-9.

[5] Guo Min. Vehicle detection technology and comparative analysis of vehicle detector[A]. Chinese Transportation Information Industry[J], 2003, (10): 55-56. 